\title{
Improving measurement accuracy and feasibility to support long-term blood pressure management
}

\author{
Kei Asayama ${ }^{1,2,3} \cdot$ Takayoshi Ohkubo ${ }^{1,3}$
}

Received: 4 December 2021 / Revised: 11 December 2021 / Accepted: 14 December 2021 / Published online: 13 January 2022

(c) The Author(s), under exclusive licence to The Japanese Society of Hypertension 2022

\section{Automated office blood pressure measurement conditions}

We measure blood pressure in individuals to assess their current condition and to predict future complications. The two independent papers regarding automated office blood pressure (AOBP) measured by the cuff-oscillometric principle that were consecutively published in Hypertension [1,2] indicate the need to pay more attention to the balance between the accuracy and feasibility of blood pressure measurement. Based on 113 diverse community-dwelling individuals (mean age, 55 years), Brady et al. [1] assessed the difference among three rest periods before AOBP, measured under the attended setting by a research nurse. Other measurement conditions were strictly uniform to follow those in the Systolic Blood Pressure Intervention Trial (SPRINT) [3], as shown in Table 1. The order of the antecedent rest was randomly assigned in this trial. The AOBP values were minimally different between the shorter $(2 \mathrm{~min})$ and no rest time groups and the group after $5 \mathrm{~min}$ of rest. Compatible and noninferior results were obtained among those with systolic blood pressure $<140 \mathrm{mmHg}$, suggesting the usefulness of shorter rest time before the first measurement in the screening setting because it minimally affects specificity but increases sensitivity with smaller time investment for the majority of people.

Juraschek et al. measured unattended AOBP in 102 patients (mean age, 59.2 years) after 1-day ambulatory blood pressure

Kei Asayama

kei@asayama.org

1 Department of Hygiene and Public Health, Teikyo University School of Medicine, Tokyo, Japan

2 Studies Coordinating Centre, Research Unit Hypertension and Cardiovascular Epidemiology, KU Leuven Department of Cardiovascular Sciences, University of Leuven, Leuven, Belgium

3 Tohoku Institute for Management of Blood Pressure, Sendai, Japan monitoring [2]. Intervals of the three consecutive AOBP measurements were set to $30 \mathrm{~s}$ for the first 51 patients over 5 months beginning in August and $60 \mathrm{~s}$ for the latter 51 patients during the following year. Visually, the distribution of AOBP was consistent among all measurements for each population. However, the second and third AOBP diastolic measurements were significantly lower than the first measurement, irrespective of the measurement intervals. All the other analyses focused on comparing AOBP with 24-h ambulatory blood pressure monitoring in each group, though adjusted for confounders. Therefore, direct comparisons between 30- and 60-s time intervals were not provided; nevertheless, the results might enable us to improve the feasibility of AOBP measurement in clinical practice by utilizing 30-s intervals instead of 60-s intervals for unattended AOBP measurement. Yarows et al. demonstrated that even a 15-s interval between readings did not produce different blood pressure values from the 60-s interval when oscillometric devices were used (difference, $-1.1 /-0.1 \mathrm{mmHg}$ in systolic/ diastolic measurement; $p=0.8 />0.9$ ) [4]. Ischemia in an arm distal to the measurement cuff can lower the recorded blood pressure values; however, ischemia from total occlusion caused by the cuff would last only $10 \mathrm{~s}$ (when measured by inflating the cuff to $20 \mathrm{mmHg}$ above the systolic pressure and using a deflation rate of $2 \mathrm{mmHg} / \mathrm{s}$ ) and thus would be unlikely to change the blood pressure values $[4,5]$. Based on auscultatory methods, venous congestion or hyperemia may raise systolic blood pressure and decrease diastolic blood pressure $[5,6]$. However, repeated auscultatory measures are reported to need intervals of at least $30 \mathrm{~s}$ [6], which is also shorter than the intervals recommended by the current guidelines for office blood pressure measurements [7, 8]. Under keeping accuracy warranted, we should aim to ensure the efficiency of office blood pressure measurements and inform patients, doctors and health care providers on how to perform active measurements in the clinical setting.

The blood pressure measurement interval is generally based on the end-to-start principle [2, 6], i.e., the measurement interval is counted from the end of complete cuff 


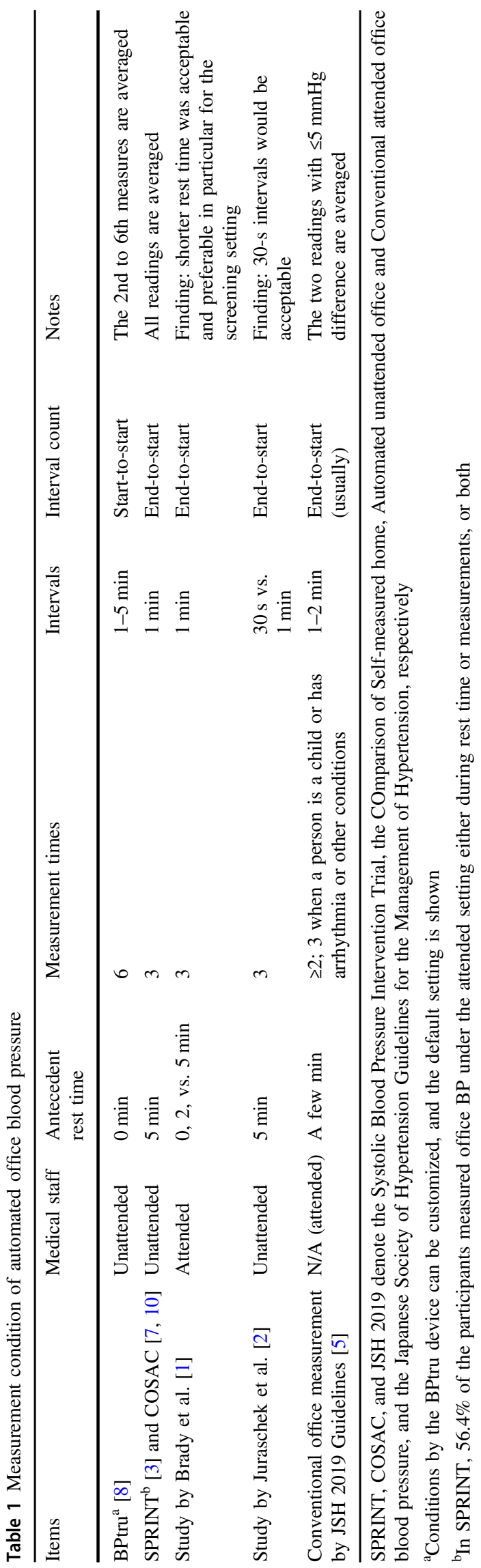

deflation of one reading to the start of the next reading [9]. When we use the AOBP device with the start-to-start principle (this setting is embedded in the BpTRU [10] by which several advantages of AOBP have been reported), interval times vary due to the different cuff inflationdeflation periods among individuals. In all the AOBP-based studies introduced in this article [1-3, 9, 11, 12], an Omron HEM-907 (Omron Healthcare, Kyoto, Japan) automated device was used, and the algorithm was the same as that used in SPRINT [3]—a 5-min wait before the first measurement was employed, and a total of three measurements with a 1-min end-to-start interval were automatically performed by the device. The feature is currently available in several automated devices from different manufacturers, e.g., Microlife WatchBP Office series (Microlife AG, Widnau, Switzerland) and A\&D TM-2441 (A\&D Company, Tokyo, Japan). In addition, some recent automated devices can capture blood pressure during the cuff inflation phase by preventing the usual loud noise that influences an accurate oscillometric signal [13]. The end-to-start principle is thus preferable for defining the intervals of blood pressure measurements, whereas the impacts of such various motions during the cuff inflation-deflation period on blood pressure values should be separately assessed.

\section{Compatibility and reproducibility of blood pressure measurement methods}

According to the aforementioned SPRINT condition for unattended AOBP measurement (Table 1) [3], the COmparison of Self-measured home, Automated unattended office and Conventional attended office blood pressure (COSAC) study was conducted to compare unattended AOBP with conventional in-office and self-measured home blood pressures. Although the average AOBP level was similar to home blood pressure in a patient population with hypertension ( $n=308$; mean age, 71.8 years), the study concluded that home blood pressure could not be replaced with AOBP because of the wide range of differences (the mean [SD] difference between AOBP and morning home, 0.9 [17.8]/-4.5 [10.5] mmHg in systolic/diastolic measurement) and low correlations $(r=0.07 / 0.53)$ in individuals [9]. Instead, AOBP was highly correlated with conventionally measured office blood pressure $(r=0.73 /$ 0.86 ), while the average level of AOBP was $10.4 / 4.2 \mathrm{mmHg}$ lower than that of conventional office measurement. Furthermore, the three consecutive AOBP readings within one occasion had high reproducibility within each patient $(r>0.90)$ [9]. These results suggest that unattended AOBP measurements can provide reliable values within a single occasion and theoretically enhance conventional office measurements. Low compatibility between AOBP 
and home blood pressure was also observed among another Japanese older patient population $(n=72$; mean age, 76.5 years) [11]. The mean (SD) differences between unattended AOBP and home blood pressure and between unattended and attended AOBP, without consideration of resting time, were $0.3(17.0) / 0.8$ (10.2) $\mathrm{mmHg}$ and $5.5(10.7) / 0.5$ (7.9) $\mathrm{mmHg}$, respectively [11]. Moreover, $22 \%$ of patients had a difference between home blood pressure and unattended AOBP of more than $20 \mathrm{mmHg}$ [11].

In the COSAC study, the long-term reproducibility of AOBP was further assessed [12]. The mean systolic blood pressure differences between baseline and 1 month later, as well as baseline and 1 year later $(n=287$; follow-up rate, 93.2\%), were less than $1.5 \mathrm{mmHg}$, whereas the standard deviations of the differences in home, AOBP, and conventional attended office measurements during the 1-year interval were 7.7, 14.5 , and $15.3 \mathrm{mmHg}$, respectively, as demonstrated in the Bland and Altman plots (Fig. 1) [12]. In-office blood pressure values were less stable than home blood pressure even when the AOBP method was switched to in-office measures. Meanwhile, the home blood pressure reproducibility based on the $r$ correlation statistic was similar to in-office blood pressure measurements [12]. Therefore, home blood pressure should be frequently assessed for the long-term management and treatment of hypertension.

\section{Further expansion of blood pressure management including in-office measurement}

We emphasize that the high reliability of AOBP [9] and better prognostic significance of home blood pressure [14] do not negate the usefulness of conventional office blood pressure, which is supported by vast evidence $[15,16]$, particularly at the phase of initializing antihypertensive drug therapy. In a home blood pressure-based individual participant data meta-analysis [14], home blood pressure exhibited improved risk stratification in general among people without antihypertensive drug treatment but did not significantly improve the prediction of death or cardiovascular outcomes in those with office blood pressure $\geq 160 / \geq 100 \mathrm{mmHg}$. Unfortunately, some patients newly diagnosed with hypertension are reluctant to use antihypertensive drugs because of their comparably low home blood pressure. Moreover, they sometimes insist on prioritizing the assessment of long-term home blood pressure measurements before drug initiation. Previous reports have demonstrated that white-coat hypertension, high office blood pressure but normal out-of-office blood pressure, is not a greater risk factor for cardiovascular diseases than masked hypertension or sustained hypertension $[14,17]$ and may mislead such patients and their responsible
Fig. 1 Bland-Altman plots for the comparison of systolic blood pressure measurements between (A) Visit 1 (baseline) and Visit 2 (1 month later) and between (B) Visit 1 and Visit 3 (1 year later). Scales of the three $Y$-axes are aligned. Mean differences and \pm 2 SD between paired measurements are represented by dotted lines, and the values of the mean (SD) difference are shown in boxes in each panel. Dash-dotted lines are placed where the difference is zero. Reproduced from Asayama et al. [12]
(A) Between Visit 1 and Visit 2
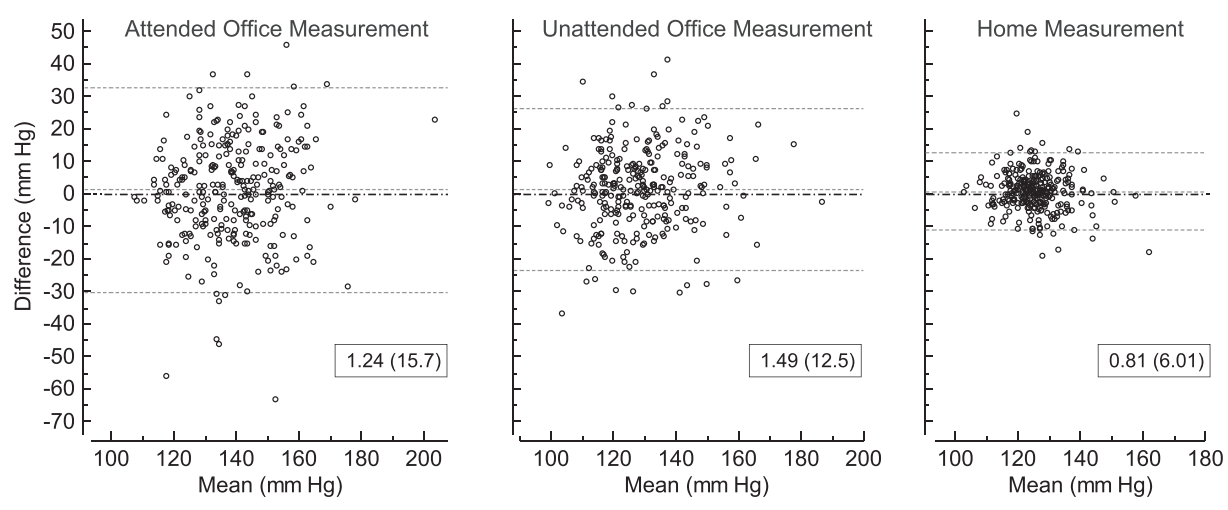

(B) Between Visit 1 and Visit 3

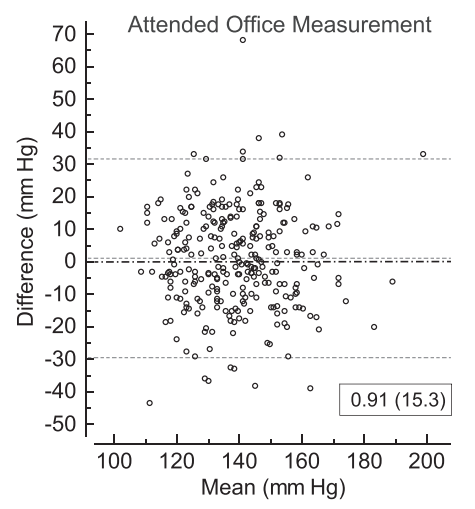

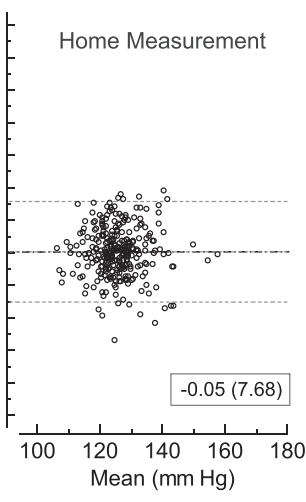


doctors. However, those with office blood pressure 160/100 $\mathrm{mmHg}$ or higher, classified as having stage/grade II/III hypertension, have very high cardiovascular disease risk regardless of age [16] and the white-coat effect. Therefore, we should not allow postponement of the start of antihypertensive drug treatment because of the lack of home blood pressure values in such high-risk patients. As per the current hypertension management guidelines in Japan [7] and Europe [8], it is crucially recommended that these patients (except those with no other risk factors according to the Japanese Guidelines [7], meaning that they are considered to have moderate risk) start antihypertensive drug therapy immediately. Nevertheless, for the long-term management of hypertension, including lifestyle modification, dietary advice, and antihypertensive drug therapy, we need to measure inoffice blood pressure as accurately as possible under feasible conditions and repeatedly assess home blood pressure. Much more should patients with low (mild) to moderate risk their blood pressure be measured. For the further expansion of blood pressure measurements, the measurement conditions should be reassessed in detail regarding accuracy and feasibility.

\section{Compliance with ethical standards}

Conflict of interest KA and TO received research support from Omron Healthcare and honoraria from Takeda Pharmaceutical.

Publisher's note Springer Nature remains neutral with regard to jurisdictional claims in published maps and institutional affiliations.

\section{References}

1. Brady TM, Charleston J, Ishigami J, Miller ER 3rd, Matsushita K, Appel LJ. Effects of different rest period durations prior to blood pressure measurement: the Best Rest Trial. Hypertension. 2021; 78:1511-9.

2. Juraschek SP, Ishak AM, Mukamal KJ, Wood JM, Anderson TS, Cohen ML, et al. Impact of 30- versus 60-second time intervals between automated office blood pressure measurements on measured blood pressure. Hypertension. 2021;78:1502-10.

3. Johnson KC, Whelton PK, Cushman WC, Cutler JA, Evans GW, Snyder JK, et al. Blood pressure measurement in SPRINT (Systolic Blood Pressure Intervention Trial). Hypertension. 2018;71:848-57.
4. Yarows SA, Patel K, Brook R. Rapid oscillometric blood pressure measurement compared to conventional oscillometric measurement. Blood Press Monit. 2001;6:145-7.

5. Eguchi K, Kuruvilla S, Ogedegbe G, Gerin W, Schwartz JE, Pickering TG. What is the optimal interval between successive home blood pressure readings using an automated oscillometric device? J Hypertens. 2009;27:1172-7.

6. Imamura M, Asayama K, Sawanoi Y, Shiga T, Saito K, Ohkubo T. Effects of measurement intervals on the values of repeated auscultatory blood pressure measurements. Clin Exp Hypertens. 2020;42:105-9.

7. Umemura S, Arima H, Arima S, Asayama K, Dohi Y, Hirooka Y, et al. The Japanese Society of Hypertension Guidelines for the Management of Hypertension (JSH 2019). Hypertens Res. 2019; 42:1235-481.

8. Williams B, Mancia G, Spiering W, Agabiti Rosei E, Azizi M, Burnier M, et al. 2018 ESC/ESH Guidelines for the management of arterial hypertension. Eur Heart J. 2018;39:3021-104.

9. Asayama K, Ohkubo T, Rakugi H, Miyakawa M, Mori H, Katsuya T, et al. Comparison of blood pressure values-self-measured at home, measured at an unattended office, and measured at a conventional attended office. Hypertens Res. 2019;42:1726-37.

10. Mattu GS, Heran BS, Wright JM. Overall accuracy of the BpTRUan automated electronic blood pressure device. Blood Press Monit. 2004;9:47-52.

11. Toba A, Ishikawa J, Suzuki A, Harada K. Automated office blood pressure measurement by elderly patients in the waiting room. Blood Press Monit. 2021;26:321-7.

12. Asayama K, Ohkubo T, Rakugi H, Miyakawa M, Mori H, Katsuya $\mathrm{T}$, et al. Direct comparison of the reproducibility of in-office and self-measured home blood pressures. J Hypertens. 2021. https://doi.org/10.1097/HJH.0000000000003026.

13. Asayama K, Fujiwara T, Hoshide S, Ohkubo T, Kario K, Stergiou GS, et al. Nocturnal blood pressure measured by home devices: evidence and perspective for clinical application. J Hypertens. 2019;37:905-16.

14. Asayama K, Thijs L, Brguljan-Hitij J, Niiranen TJ, Hozawa A, Boggia J, et al. Risk stratification by self-measured home blood pressure across categories of conventional blood pressure: a participant-level meta-analysis. PLoS Med. 2014;11:e1001591.

15. Staessen JA, Wang JG, Thijs L. Cardiovascular protection and blood pressure reduction: a meta-analysis. Lancet. 2001;358: $1305-15$.

16. Lewington S, Clarke R, Qizilbash N, Peto R, Collins R. Agespecific relevance of usual blood pressure to vascular mortality: a meta-analysis of individual data for one million adults in 61 prospective studies. Lancet. 2002;360:1903-13.

17. Fujiwara T, Matsumoto C, Asayama K, Ohkubo T, Hoshide S. Are the cardiovascular outcomes of participants with white-coat hypertension poor compared to those of participants with normotension? A systemic review and meta-analysis. Hypertens Res. 2019;42:825-33. 